\title{
Implementation of a Process Analytical Technology (PAT) System in a Freeze Drying Process Using Raman Spectroscopy for In-Line Process Monitoring
}

T.R.M. De Beer ${ }^{*}{ }^{1}$, M. Alles $\varnothing^{2}$, F. Goethals ${ }^{3}$, A. Coppens ${ }^{1}$, Y. Vander Heyden ${ }^{4}$, H. Lopez de

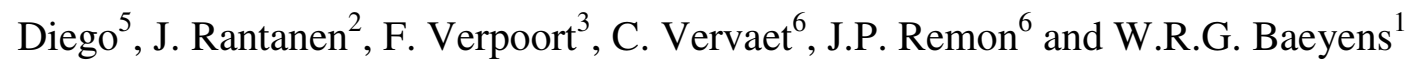

\section{SUPPORTING INFORMATION}

${ }^{1}$ Laboratory of Drug Quality Control, Department of Pharmaceutical Analysis, Ghent University, Harelbekestraat 72, B-9000 Ghent, Belgium

${ }^{2}$ Department of Pharmaceutics and Analytical Chemistry, Faculty of Pharmaceutical Sciences, University of Copenhagen, Universitetsparken 2, DK-2100 Copenhagen, Denmark

${ }^{3}$ Department of Inorganic and Physical Chemistry, Ghent University, Krijgslaan 281 (S3), B9000 Ghent, Belgium

${ }^{4}$ Department of Pharmaceutical and Biomedical Analysis, Pharmaceutical Institute, Vrije Universiteit Brussel - VUB, Laarbeeklaan 103, B-1090 Brussels, Belgium ${ }^{5}$ Analytical R \& D, H. Lundbeck A/S, DK-2500 Valby, Denmark

${ }^{6}$ Laboratory of Pharmaceutical Technology, Ghent University, Harelbekestraat 72, B-9000 Ghent, Belgium

Supporting Information Figures: 7 (figure S-1 to figure S-7)

Supporting Information Tables: 3 (table S-1 to table S-3) 
Figure S-1. Experimental setup for the in-line and real-time monitoring of freeze drying processes using Raman spectroscopy

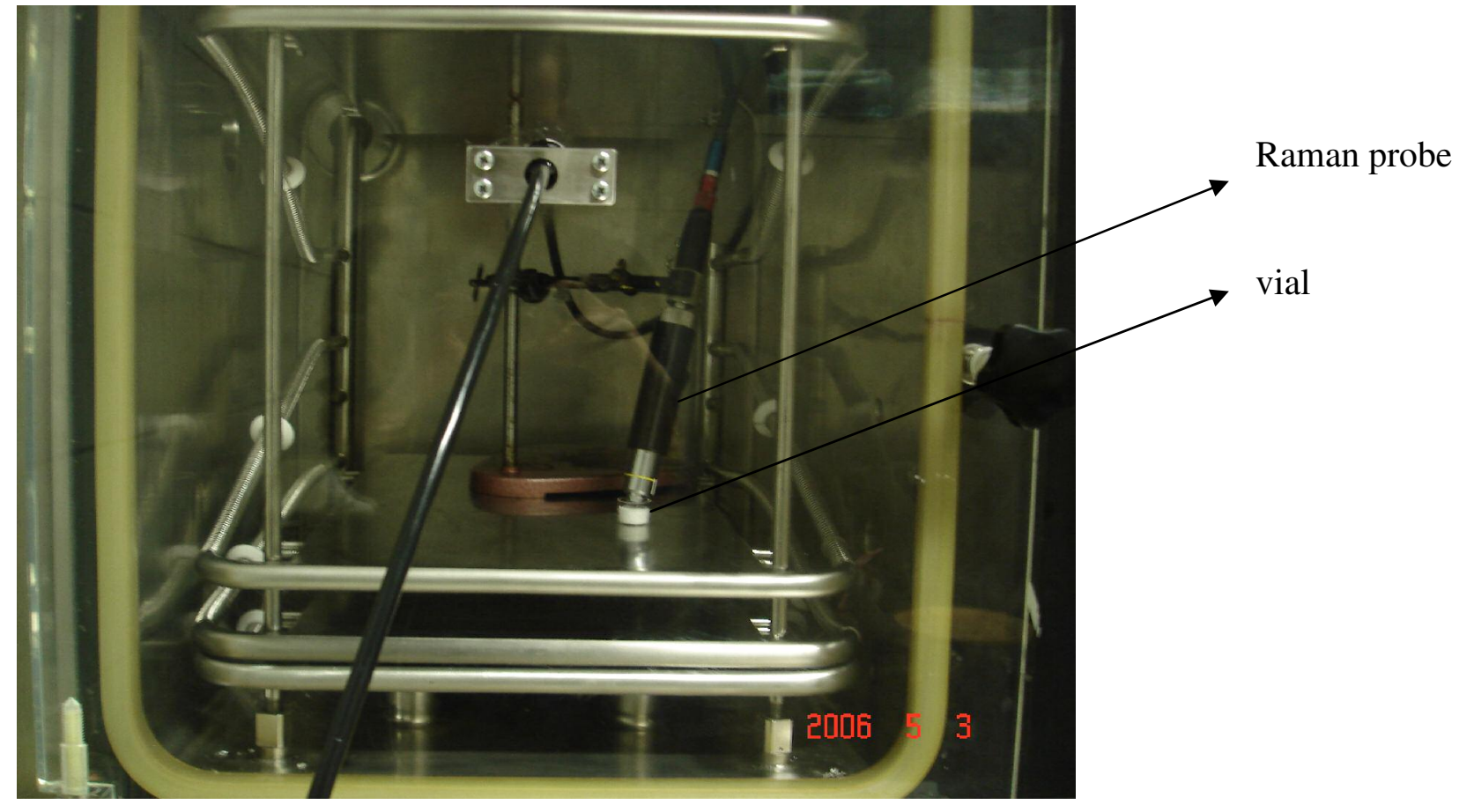


Figure S-2. Raman spectra of mannitol recorded during the freezing stage of a freeze drying process (experiment 1)

Figure S-2a: spectra 1-36

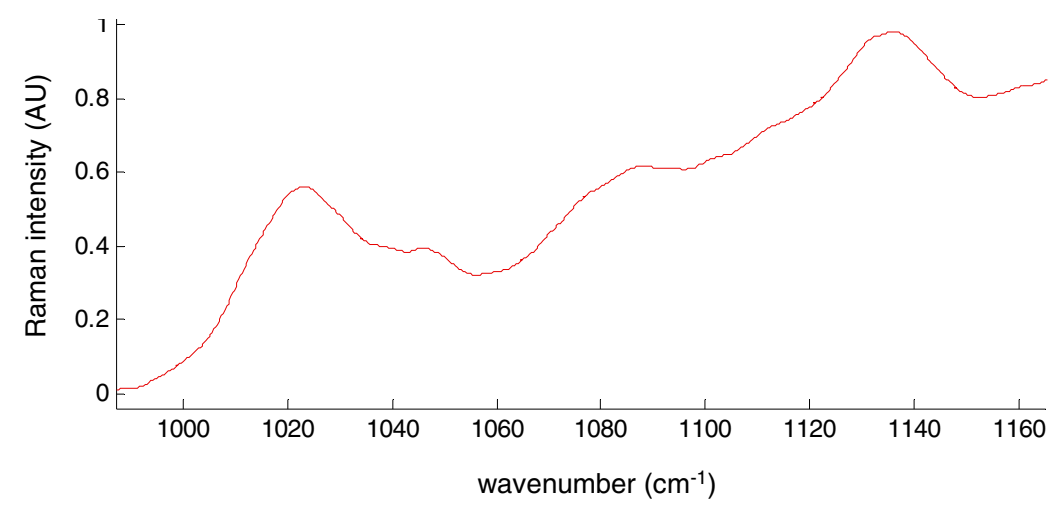

Figure S-2b: spectra 37-51

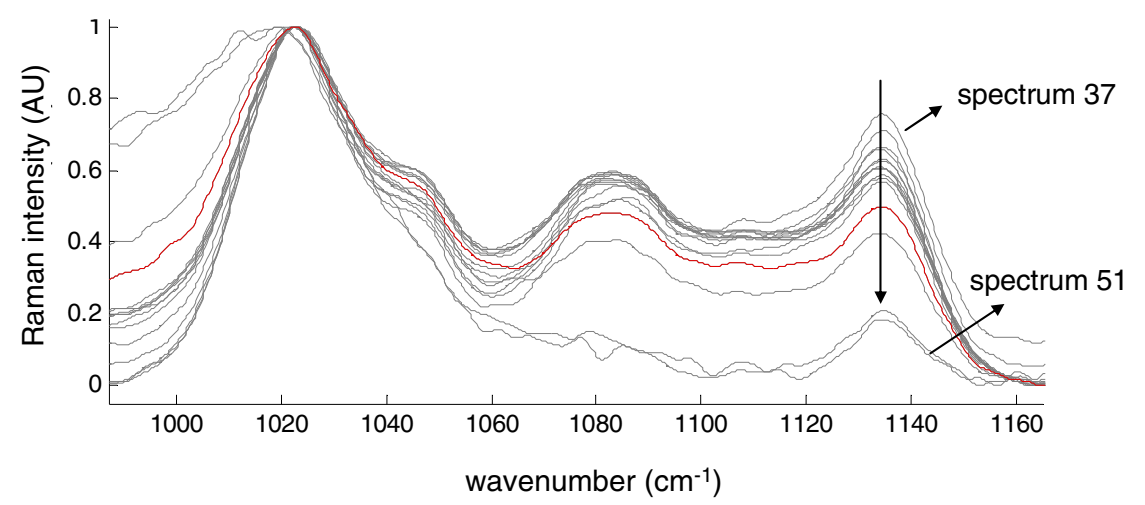

Figure S-2c: spectra 51-61

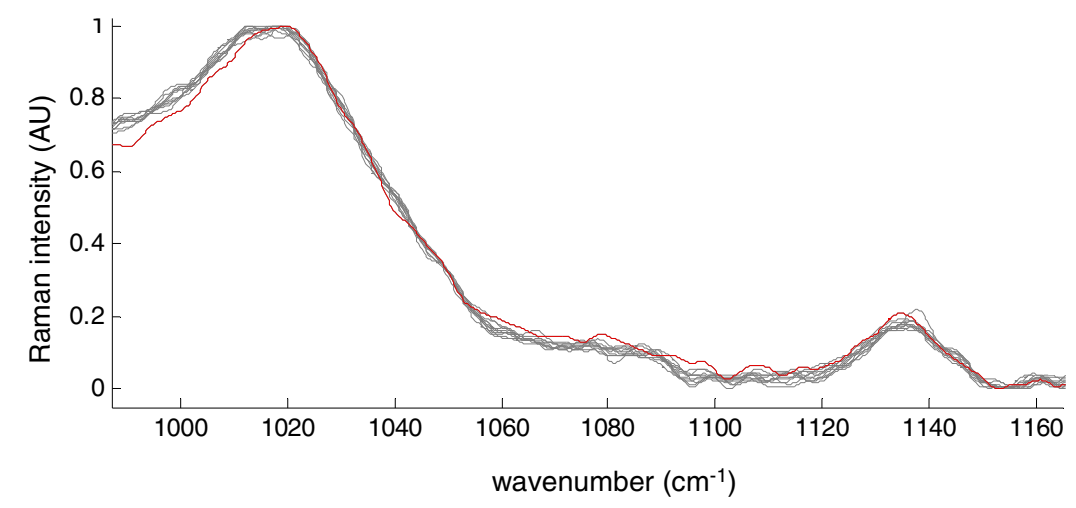


Figure S-2d: spectra 62-106

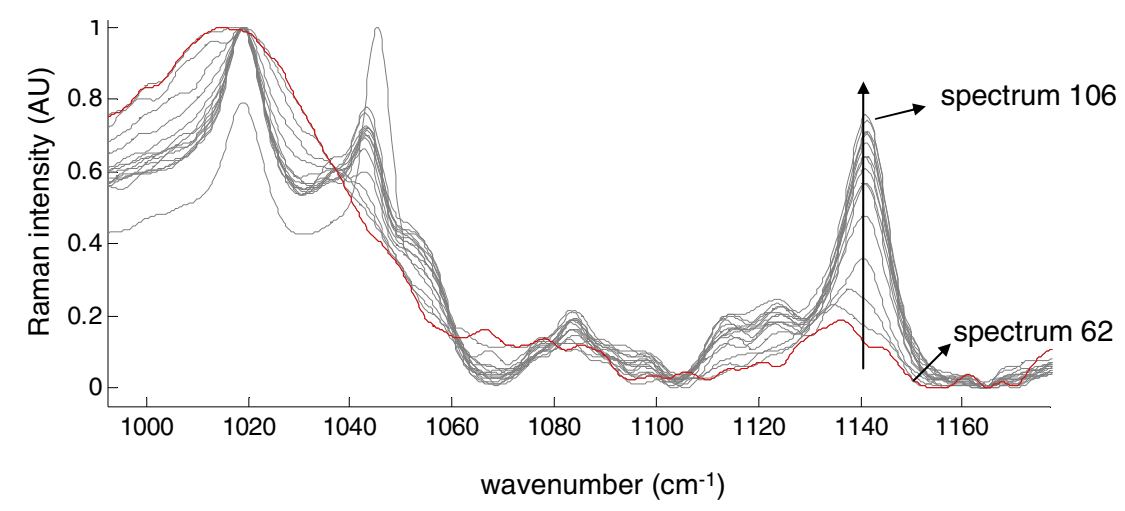

Figure S-2e: spectra 107-175

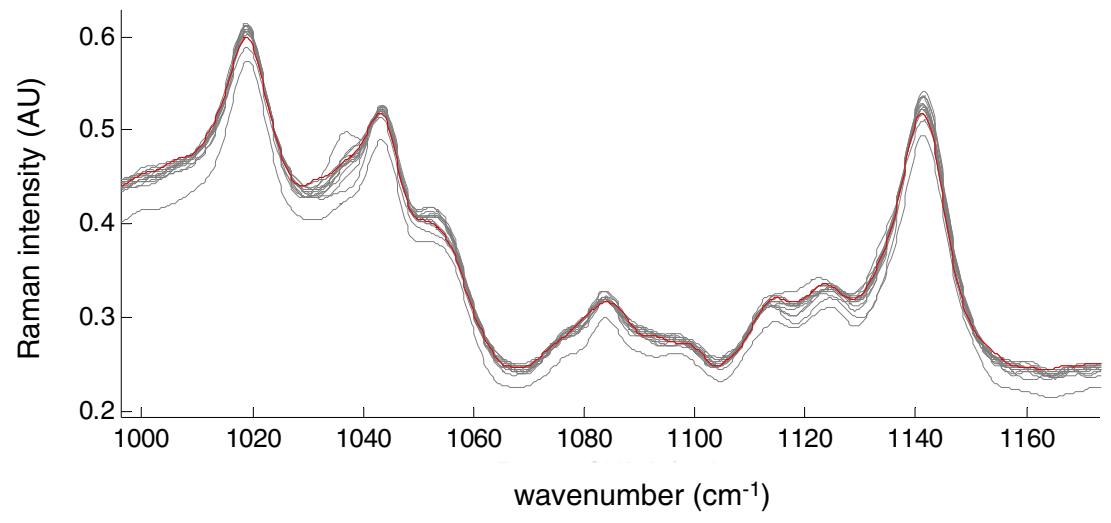




\section{Figure $S$-3.}

Fig. S-3a: Ice produces Raman signal at $215 \mathrm{~cm}^{-1}$

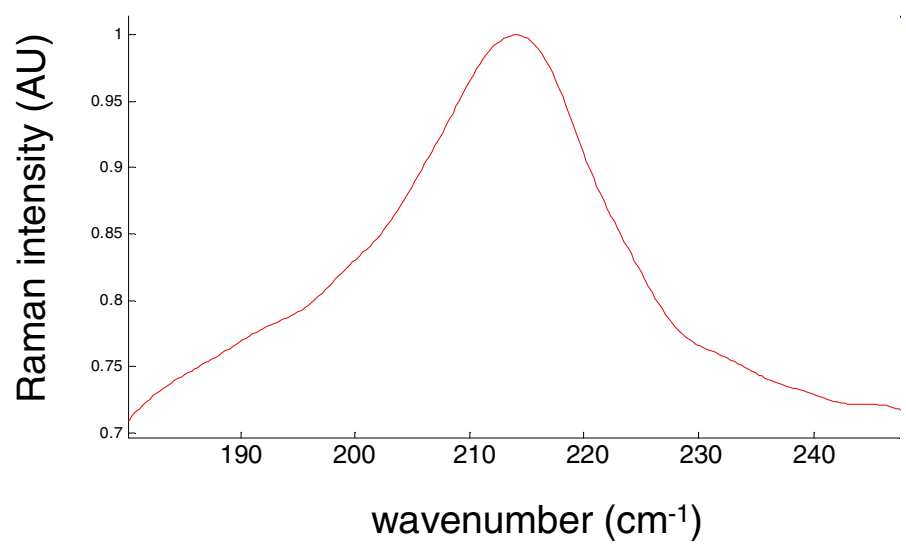

Fig. S-3b: peak intensity ice band vs. process time

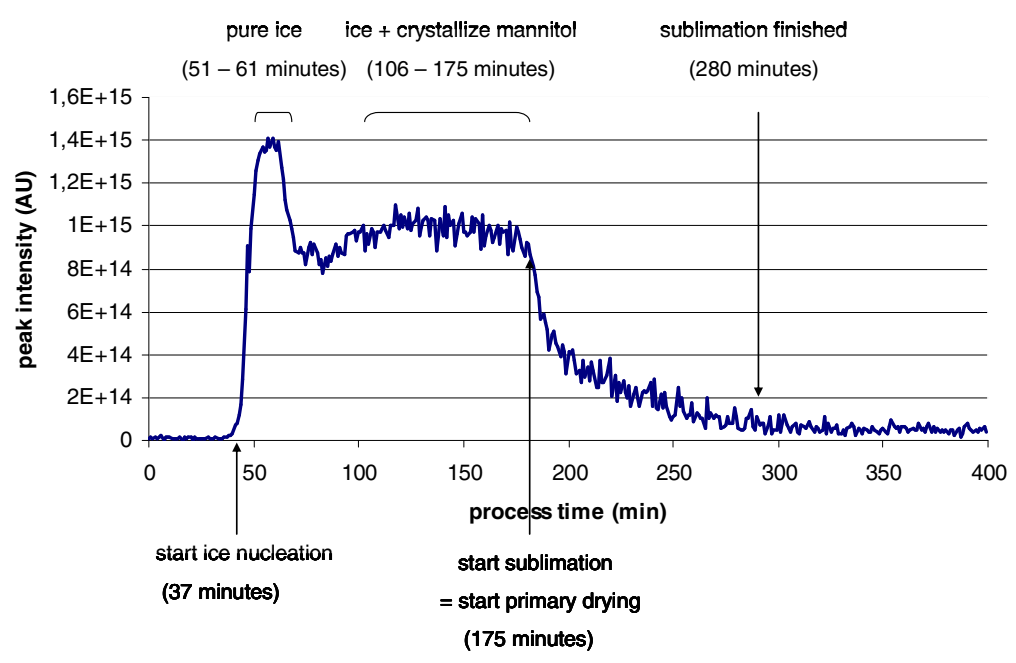


Figure S-4. NIR spectral transformations during ice sublimation

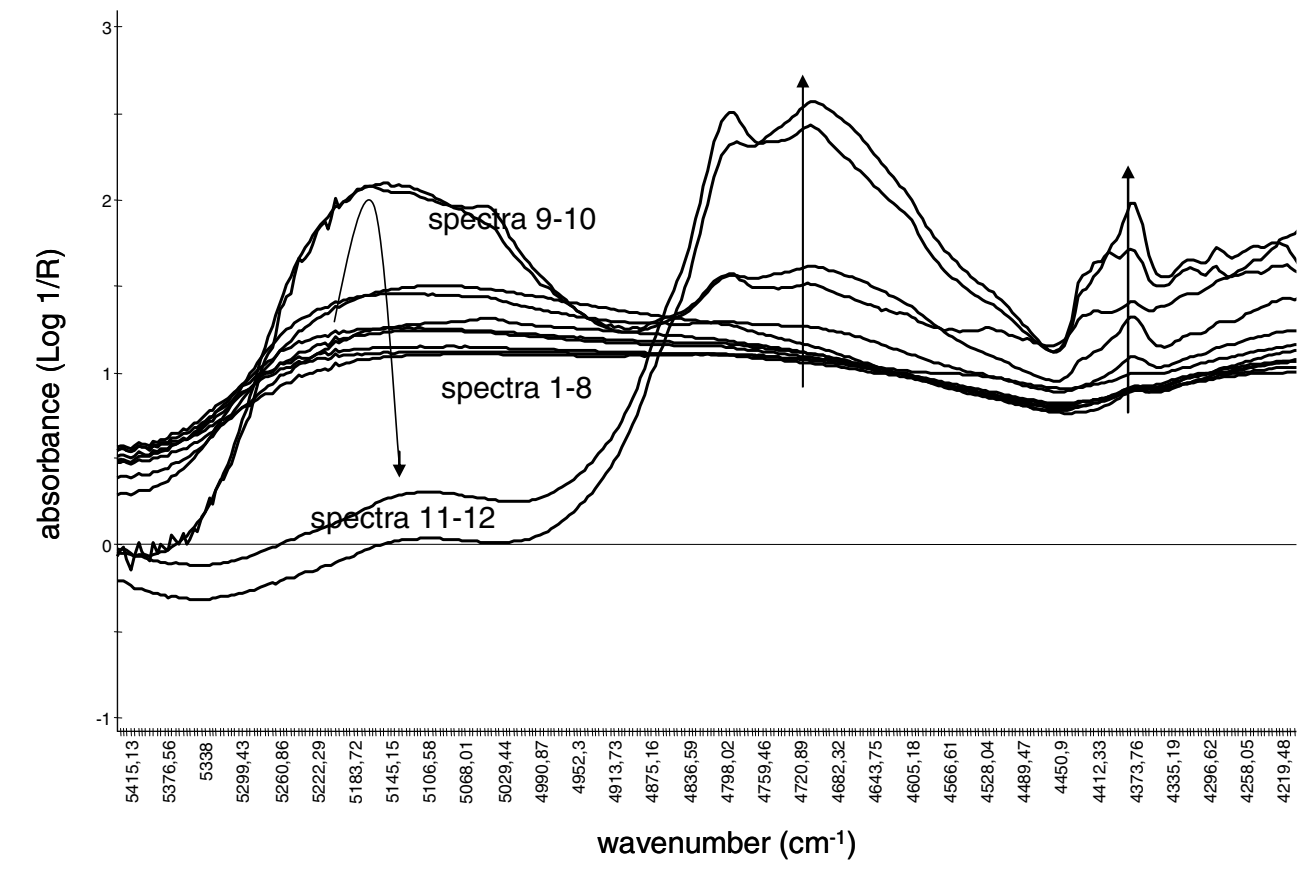


Figure S-5. Raman spectral transformation to $\alpha$-mannitol during secondary drying at $30^{\circ} \mathrm{C}$ in experiment 9

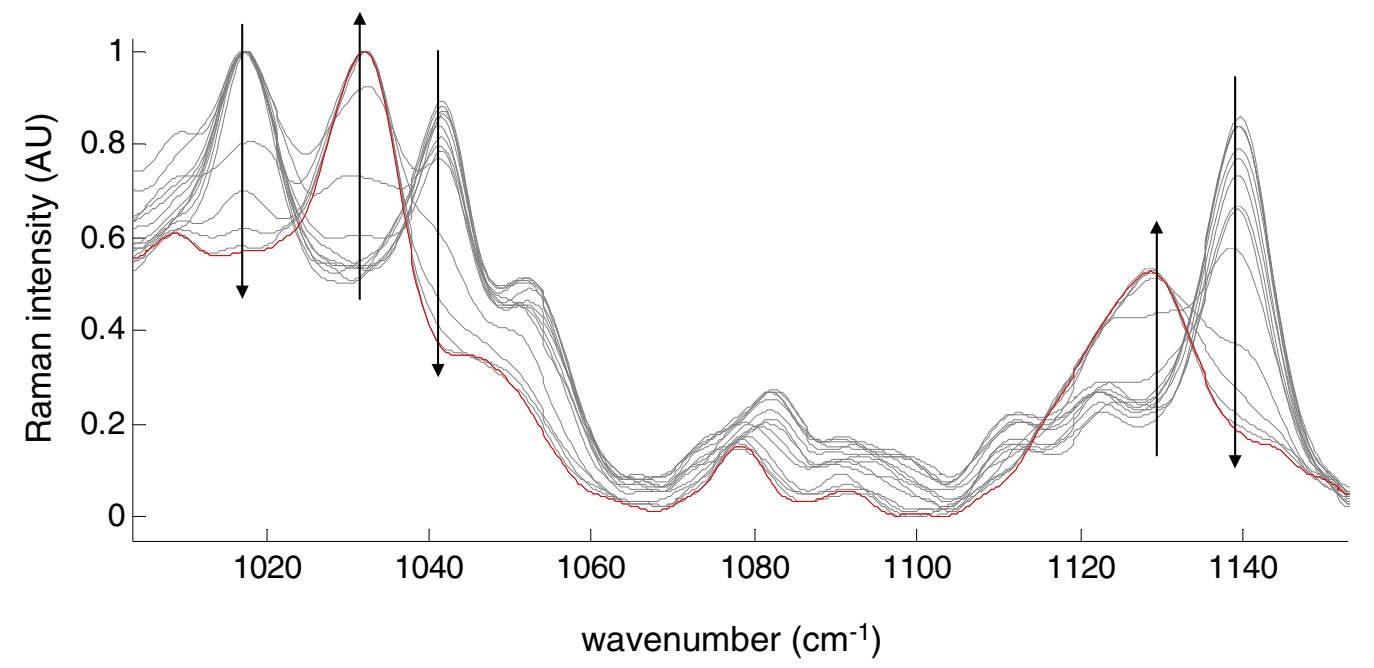


Figure S-6. Standardized Pareto chart for the response variable 'onset ice nucleation' $(\alpha=$ $0.05)$

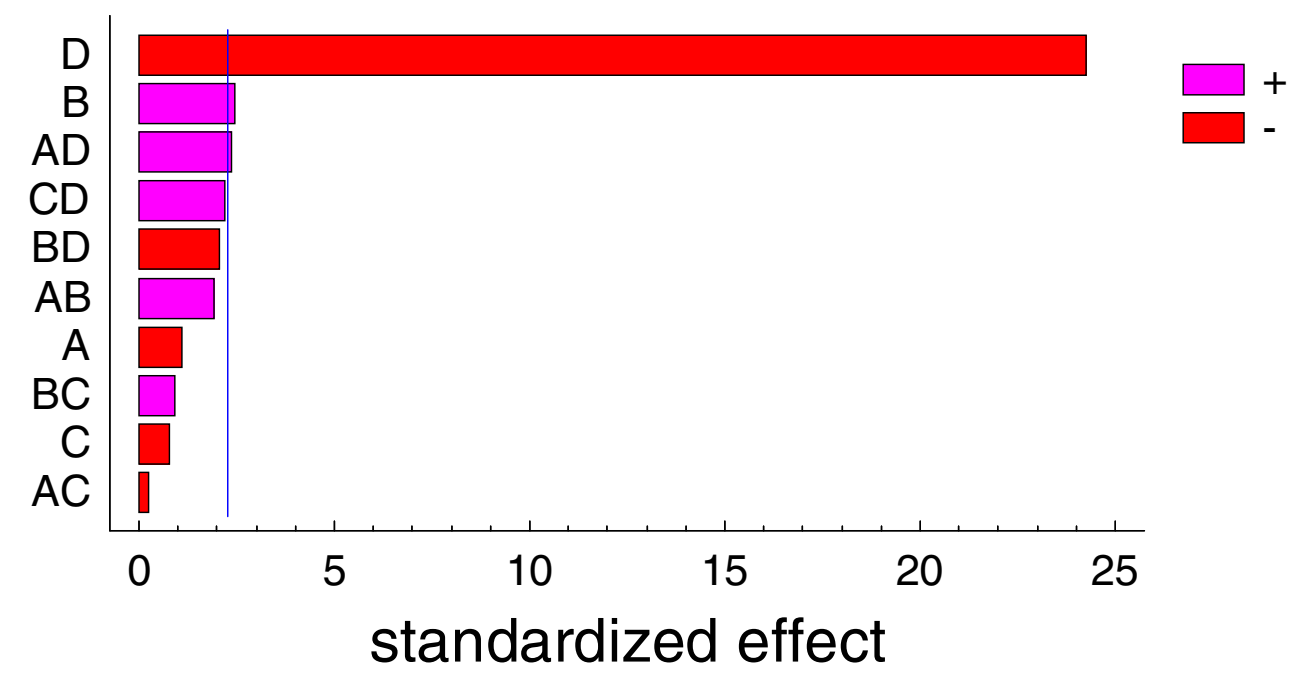

$$
\begin{aligned}
& A=\text { concentration of mannitol } \\
& B=\text { concentration of } \mathrm{NaCl} \\
& C=\text { volume } \\
& D=\text { freezing rate }
\end{aligned}
$$


Figure S-7. Screening design analysis. The units of the y-axis in all plots are minutes.

Figure S-7a: duration of ice crystallization process

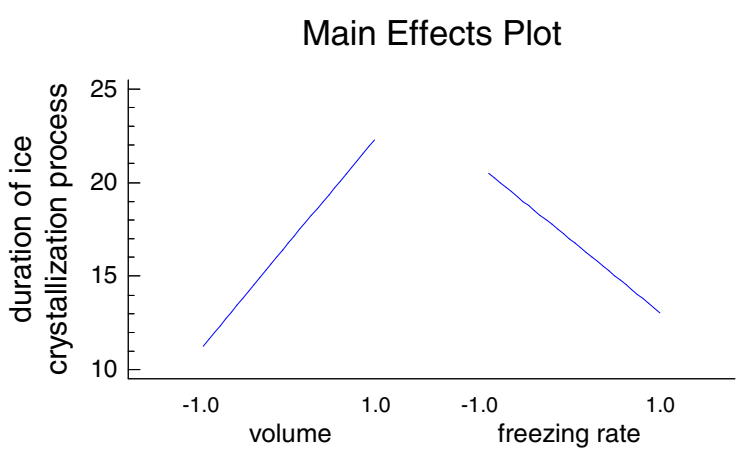

Figure S-7b: onset of mannitol crystallization
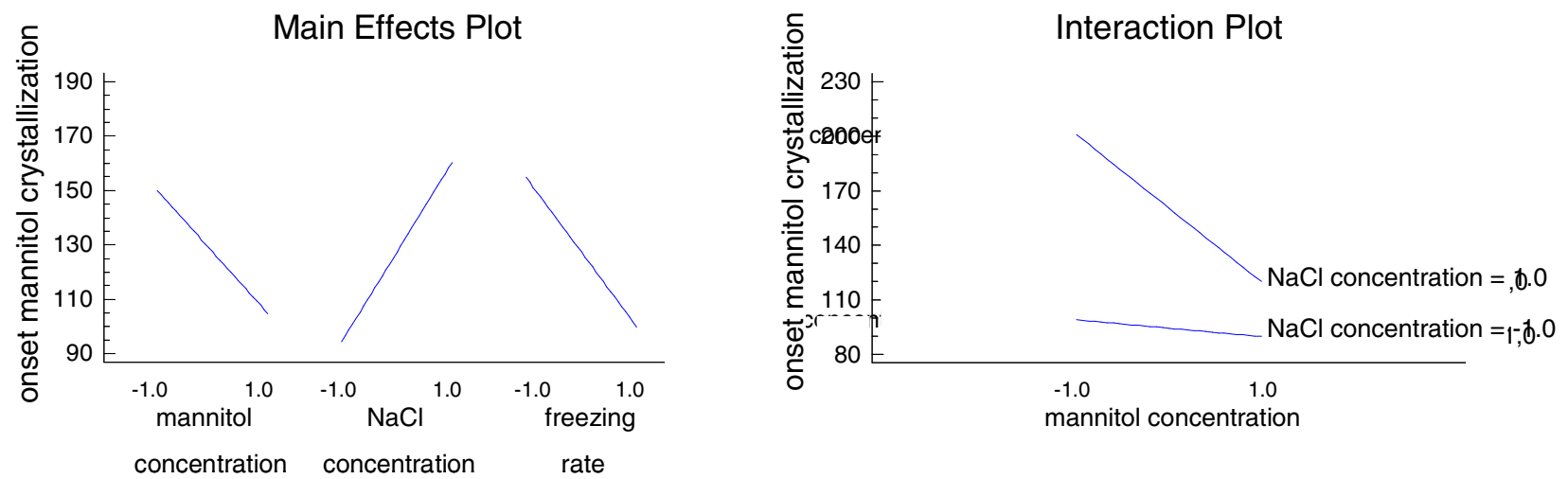

Figure S-7c: duration of mannitol crystallization process

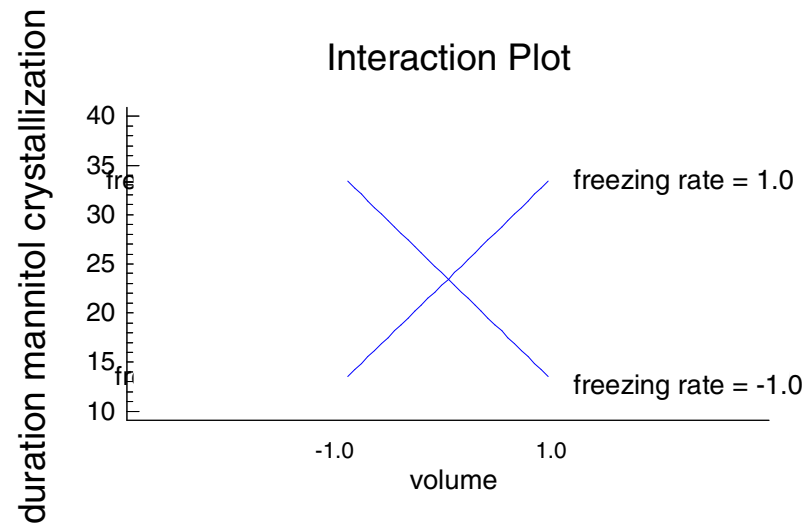


Figure S-7d: duration of sublimation process

\section{Main Effects Plot}

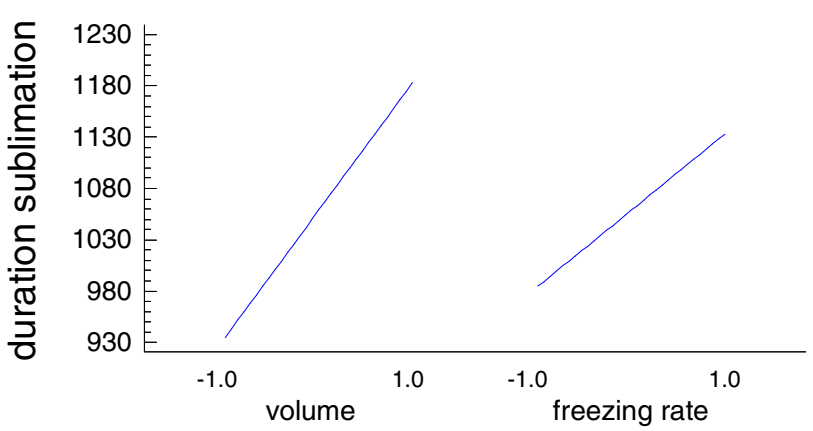

Figure S-7e: duration of amorphous mannitol state
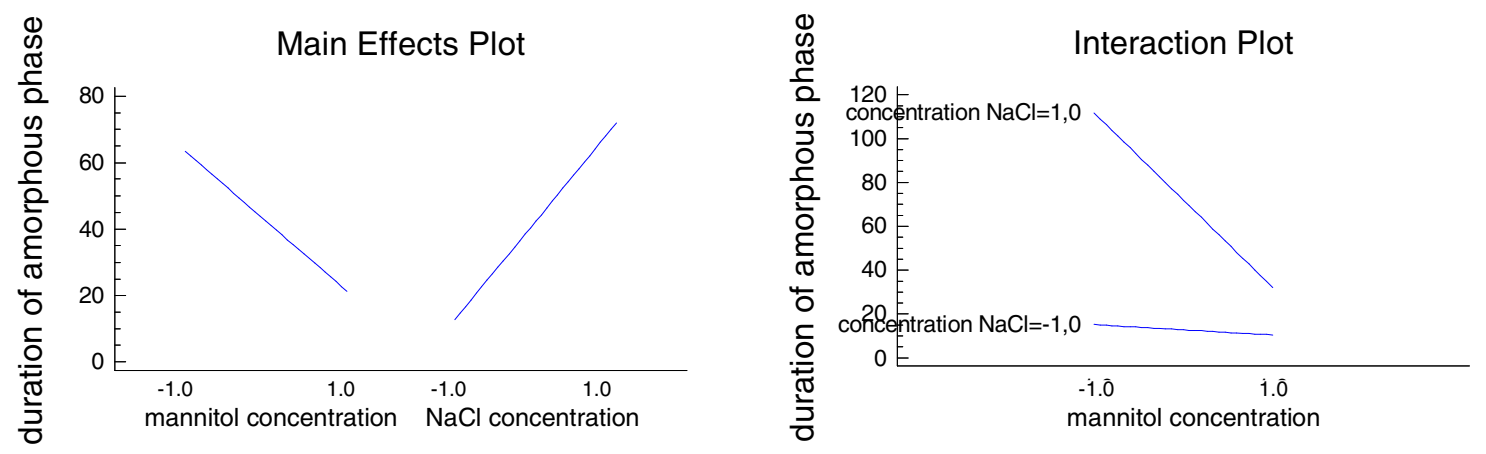
Table S-1. Freeze drying programs (fast and slow freezing) used for the experimental design experiments

\begin{tabular}{|c|c|c|c|c|c|}
\hline \multirow[t]{2}{*}{ process phase } & \multicolumn{2}{|c|}{ time (min) } & $\begin{array}{r}\text { temperatu } \\
\text { freeze d }\end{array}$ & $\begin{array}{l}\text { shelves in } \\
\text { ier }\left({ }^{\circ} \mathrm{C}\right)\end{array}$ & \multirow{2}{*}{$\begin{array}{l}\text { pressure in freeze } \\
\text { drier (mbar) }\end{array}$} \\
\hline & slow freezing & fast freezing & slow freezing & fast freezing & \\
\hline \multirow{6}{*}{ freezing } & 0 & 0 & 20 & 20 & 1000 \\
\hline & 5 & 5 & 2 & 2 & 1000 \\
\hline & 25 & 25 & -5 & -5 & 1000 \\
\hline & 120 & 30 & -25 & -45 & 1000 \\
\hline & 145 & 145 & -40 & -45 & 1000 \\
\hline & 165 & 165 & -45 & -45 & 1000 \\
\hline primary drying & 175 & 175 & -15 & -15 & 0.8 \\
\hline \multirow{2}{*}{ secondary drying } & 977 & 977 & 0 & 0 & 0.1 \\
\hline & 1097 & 1097 & 10 & 10 & 0.1 \\
\hline program end & 1517 & 1517 & 10 & 10 & 0.1 \\
\hline
\end{tabular}


Table S-2. Overview of screening design experiments. The experiments in bold were repeated one time.

\begin{tabular}{|c|c|c|c|c|}
\hline $\begin{array}{c}\text { experiment } \\
\text { number }\end{array}$ & $\begin{array}{c}\text { mannitol } \\
\text { concentration }(\%)\end{array}$ & $\begin{array}{c}\mathrm{NaCl} \\
\text { concentration }(\%)\end{array}$ & volume $(\mathrm{ml})$ & freezing rate \\
\hline 1 & 5 & $\mathbf{0}$ & 4 & fast \\
\hline 2 & 15 & 0 & 4 & slow \\
\hline 3 & 5 & 2 & 4 & slow \\
\hline 4 & 15 & 2 & 2 & slow \\
\hline 5 & 15 & 2 & 4 & slow \\
\hline 6 & 15 & $\mathbf{0}$ & 4 & fast \\
\hline 7 & 5 & $\mathbf{0}$ & 4 & slow \\
\hline 8 & 15 & 2 & 4 & fast \\
\hline 9 & 5 & 0 & 2 & slow \\
\hline 10 & 15 & 0 & 2 & slow \\
\hline 11 & 15 & 0 & 2 & fast \\
\hline 12 & 5 & 0 & 2 & fast \\
\hline 13 & 5 & 2 & 2 & fast \\
\hline 14 & 5 & 2 & 2 & slow \\
\hline 15 & 5 & 2 & 4 & fast \\
\hline 16 & 15 & 2 & 2 & fast \\
\hline
\end{tabular}


Table S-3. Overview of the mannitol solid states in the end products from all screening design experiments

\begin{tabular}{|c|c|c|}
\hline 1 & $\beta+$ hemi-hydrate & $\beta+\delta+$ hemi-hydrate \\
\hline 2 & $\delta+$ hemi-hydrate & $\beta+$ hemi-hydrate \\
\hline 3 & $\beta+\delta+$ hemi-hydrate & $\beta+\delta+$ hemi-hydrate \\
\hline 4 & $\beta$ & $\delta+$ hemi-hydrate \\
\hline 5 & $\beta+$ hemi-hydrate & $\beta+\delta+$ hemi-hydrate \\
\hline 6 & $\beta+$ hemi-hydrate & $\beta+$ hemi-hydrate \\
\hline 7 & hemi-hydrate & hemi-hydrate \\
\hline 8 & $\beta+$ hemi-hydrate & $\delta+$ hemi-hydrate \\
\hline 9 & hemi-hydrate & hemi-hydrate \\
\hline 10 & hemi-hydrate & $\beta+$ hemi-hydrate \\
\hline 11 & $\beta+$ hemi-hydrate & $\beta+\delta+$ hemi-hydrate \\
\hline 12 & $\beta+\delta+$ hemi-hydrate & $\beta+\delta+$ hemi-hydrate \\
\hline 13 & $\delta+$ hemi-hydrate & $\delta+$ hemi-hydrate \\
\hline 14 & $\beta+\delta$ & $\alpha+\delta+$ hemi-hydrate \\
\hline 15 & $\delta$ & $\beta+\delta+$ hemi-hydrate \\
\hline 16 & $\beta$ & $\beta+\delta+$ hemi-hydrate \\
\hline $6 \mathrm{BIS}$ & $\beta+$ hemi-hydrate & $\beta+$ hemi-hydrate \\
\hline $7 \mathrm{BIS}$ & hemi-hydrate & hemi-hydrate \\
\hline $1 \mathrm{BIS}$ & $\beta+\delta+$ hemi-hydrate & $\beta+\delta+$ hemi-hydrate \\
\hline $8 \mathrm{BIS}$ & $\beta+$ hemi-hydrate & $\beta+\delta+$ hemi-hydrate \\
\hline
\end{tabular}

